\title{
Assistência pré-natal multimodal com orientação integrativa: a experiência de 10 anos do Núcleo de Medicina Antroposófica da Universidade Federal de São Paulo
}

Multimodal prenatal care with integrative orientation: the 10-year experience of the Anthroposophic Medicine Center of the Federal University of São Paulo

Asistencia prenatal multimodal con orientación integrativa: la experiencia de 10 años del Núcleo de Medicina Antroposófica de la Universidad Federal de São Paulo

Mary Uchiyama Nakamura ${ }^{1}$; Jorge Kioshi Hosomi ${ }^{2}$; Moacyr Mendes Morais ${ }^{3}$; Eliane Follador ${ }^{4}$; Iracema Almeida Benevides ${ }^{5}$

\section{Resumo}

A implantação de Política Nacional de Práticas Integrativas e Complementares (PNPIC), no Sistema Único de Saúde (SUS), vem de encontro à transdisciplinaridade, quando se pretende oferecer saúde à população de uma forma multimodal, como ocorre na Medicina Antroposófica, com olhar integrativo entre os diversos profissionais. A atuação no tripé ensino-assistência-pesquisa da universidade pode ser favorável à maior promoção e harmonização entre as disciplinas, durante a assistência pré-natal, resultando na gestante mais preparada para o trabalho de parto.

Descritores: Medicina Integrativa; Terapias Complementares; Cuidado Pré-Natal; Antroposofia.

\begin{abstract}
The implantation of the National Politic of Complementary and Integrative Practices (PNPIC) in the Brazilian Unified Health System contributes to transdisciplinarity as long as it searches to offer health to the population in a multimodal way, as it happens in the Anthroposophic Medicine model, an integrative multiprofessional approach involving several professionals. The university triple way composed by teaching-assistance-extension contributes for improving the integration and harmonization among the disciplines during the prenatal care, resulting in a pregnant woman better prepared to the labor.
\end{abstract}

Key-words: Integrative Medicine; Complementary Therapies; Prenatal Care; Anthroposophy.

\section{Resumen}

La implantación de la Política Nacional de Prácticas Integrativas y Complementarias (PNPIC), en el Sistema Único de Salud (SUS), viene a alcanzar la transdisciplinariedad, cuando se pretende ofrecer salud a la población de una forma multimodal, como ocurre en la Medicina Antroposófica, con una

\footnotetext{
${ }^{1}$ Médica. Professora Titular, livre docente da Universidade Federal de São Paulo (UNIFESP). Rua Napoleão de Barros, 875. Vila Clementino, São Paulo, SP. CEP: 04024-002. E-mail: mary.uchiyama@ig.com.br

${ }^{2}$ Médico. Docente do curso de especialização “Antroposofia na saúde” na Universidade Federal de São Paulo (UNIFESP).

${ }^{3}$ Psicólogo. Docente do curso de especialização “Antroposofia na saúde” na Universidade Federal de São Paulo (UNIFESP).

${ }^{4}$ Médica. Coordenadora científica da Associação Brasileira de Medicina Antroposófica (ABMA).

${ }^{5}$ Médica. Presidente da Associação Brasileira de Medicina Antroposófica (ABMA).
} 
ISSN 2179-6750

mirada integrativa entre los diversos profesionales. La actuación en el trípode enseñanza-asistenciainvestigación de la universidad puede ser favorable a la mayor promoción y armonización entre las disciplinas, durante la asistencia prenatal, resultando en la gestante más preparada para el trabajo de parto.

Palabras-claves: Medicina Integral; Terapias Complementarias; Atención Prenatal; Antroposofía.

\section{Introdução}

A Política Nacional de Práticas Integrativas e Complementares (PNPIC), foi aprovada pelo Ministério da Saúde em 2006 e, após uma década de sua implantação no SUS, constata-se que a temática ainda não é priorizada pelos gestores, considerando-se o baixo incentivo financeiro, o baixo investimento em formação e em avaliação e monitoramento, sobretudo quanto à inserção das práticas integrativas e complementares na atenção primária à saúde ${ }^{1}$. A despeito desse panorama, tem sido observado crescente demanda da população pelo acesso e ampliação das "Práticas Integrativas e Complementares em Saúde - PICS", expressão que abarca uma grande variedade de abordagens do campo. .A Organização Mundial da Saúde (OMS) ${ }^{2,3}$, desde a década de 1970, com a criação da área temática Medicina Tradicional/Medicina Complementar e Alternativa (Traditional Medicine/Complementary and Alternative Medicine - TM/CAM), desenvolve estratégias para incentivar e orientar os países membros quanto à implantação, ampliando o acesso da população a essas práticas ${ }^{2}$. Muitas dessas modalidades estão presentes há muitas décadas, em muitos continentes como Europa, América do Norte e África e entende-se que mereçam crédito pelo fato de ser milenarmente utilizadas pela população, em diferentes culturas. Documentos mais recentes da OMS reforçam a necessidade dos governos regularem essas práticas, seus praticantes e os produtos, garantindo acesso de maneira segura, respeitosa e efetiva ${ }^{3}$ Assim, estudiosos entendem que a Medicina Tradicional e Complementar pode contribuir para a integralidade da atenção, por meio de seus distintos saberes e valores, sobretudo das racionalidades médicas vitalistas e suas diversas técnicas heterônomas e autônomas. Seus recursos enriquecedores das experiências de cuidado podem ser úteis no manejo da excessiva biomedicalização e na redução de danos ${ }^{4}$. Nas portarias originais da PNPIC, a GM nº 971 (03/2006) e no 1600 (07/2006), a Política Nacional de Práticas Integrativas e Complementares incluiu a homeopatia, medicina tradicional chinesa (acupuntura), plantas medicinais/fitoterapia, termalismo social, crenoterapia e medicina antroposófica (MA) ${ }^{5}$. Em março de 2017 a portaria GM nº 849 integrou outras 14 novas práticas ao SUS ${ }^{6}$ (Figuras 1 e 2).

Com relação à formação e capacitação dos profissionais de saúde para atuar nas práticas complementares, é importante salientar que deles são exigidos educação continuada e permanente, para poderem usar suas expertises de forma competente: a superficialidade das atividades 
ISSN 2179-6750

educativas introdutórias pode comprometer o cuidado com a saúde da população. Para isso, a formação deve ser consistente ${ }^{1}$. As universidades federais oferecem disciplinas eletivas, em cursos de graduação, especialização e residência médica em algumas das modalidades da MTC, mas majoritariamente, a formação é feita por instituições privadas ${ }^{7}$. Essa pode ser uma das razões que expliquem a escassez de oferta assistencial de MTC no mercado: os cursos teóricos gratuitos são apenas introdutórios, e os cursos longos, de maior profundidade e com maior percentual de aulas práticas, têm um custo financeiro pouco acessível. Assim, Sousa et al. ${ }^{7}$ afirmam que é estratégico tirar proveito das experiências existentes, diretrizes orientadoras da inserção da Medicina Tradicional e Complementar na Estratégia Saúde da Família - ESF/atenção primária à saúde. Ou seja, estas iniciativas deveriam ser estudadas e valorizadas, com geração de maior visibilidade, aprendizado institucional e político (Figura 3).

Figura 1. Número de consultas por tipo de PICS, Brasil, 2015

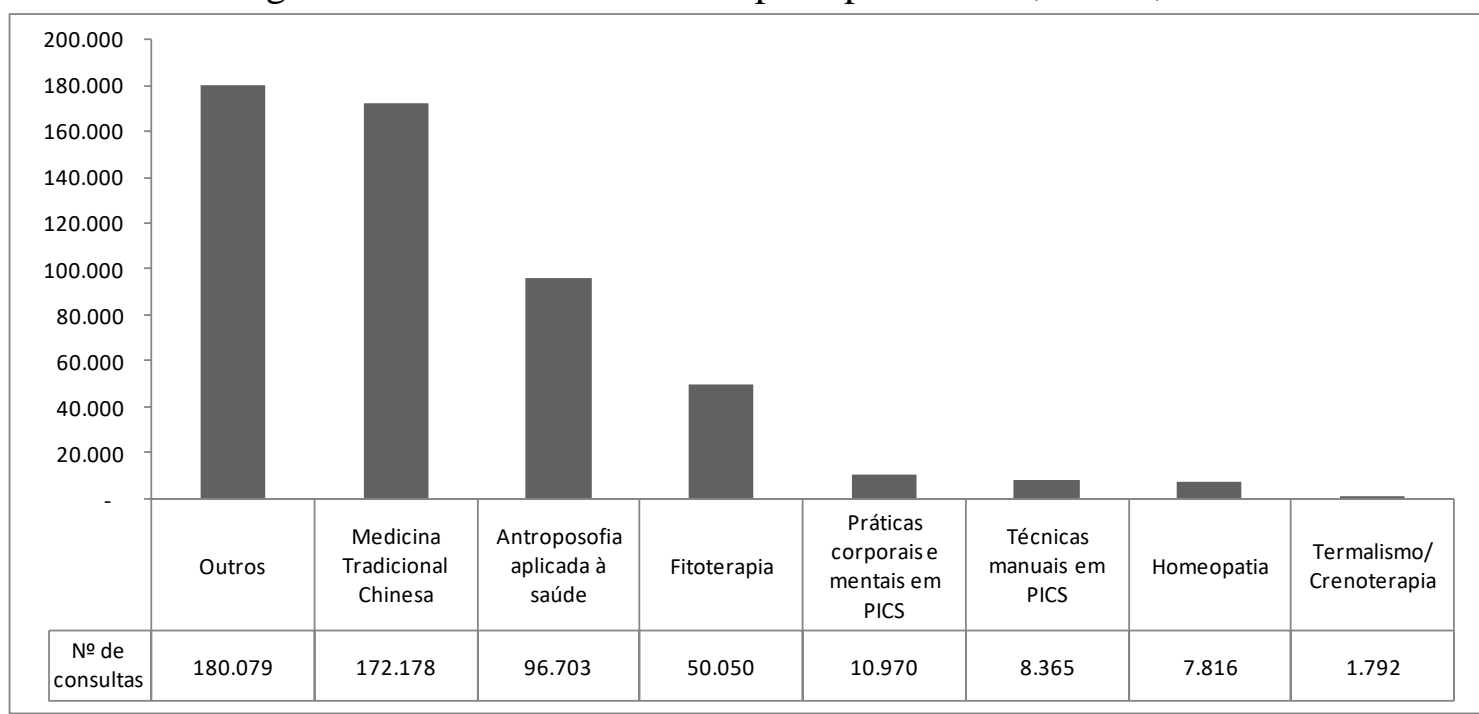

Fonte: e-SUS, DAB/SAS/MS (2015). 
ISSN 2179-6750

Figura 2. Número de atendimentos por tipo de PICS, Brasil, janeiro a agosto de 2016

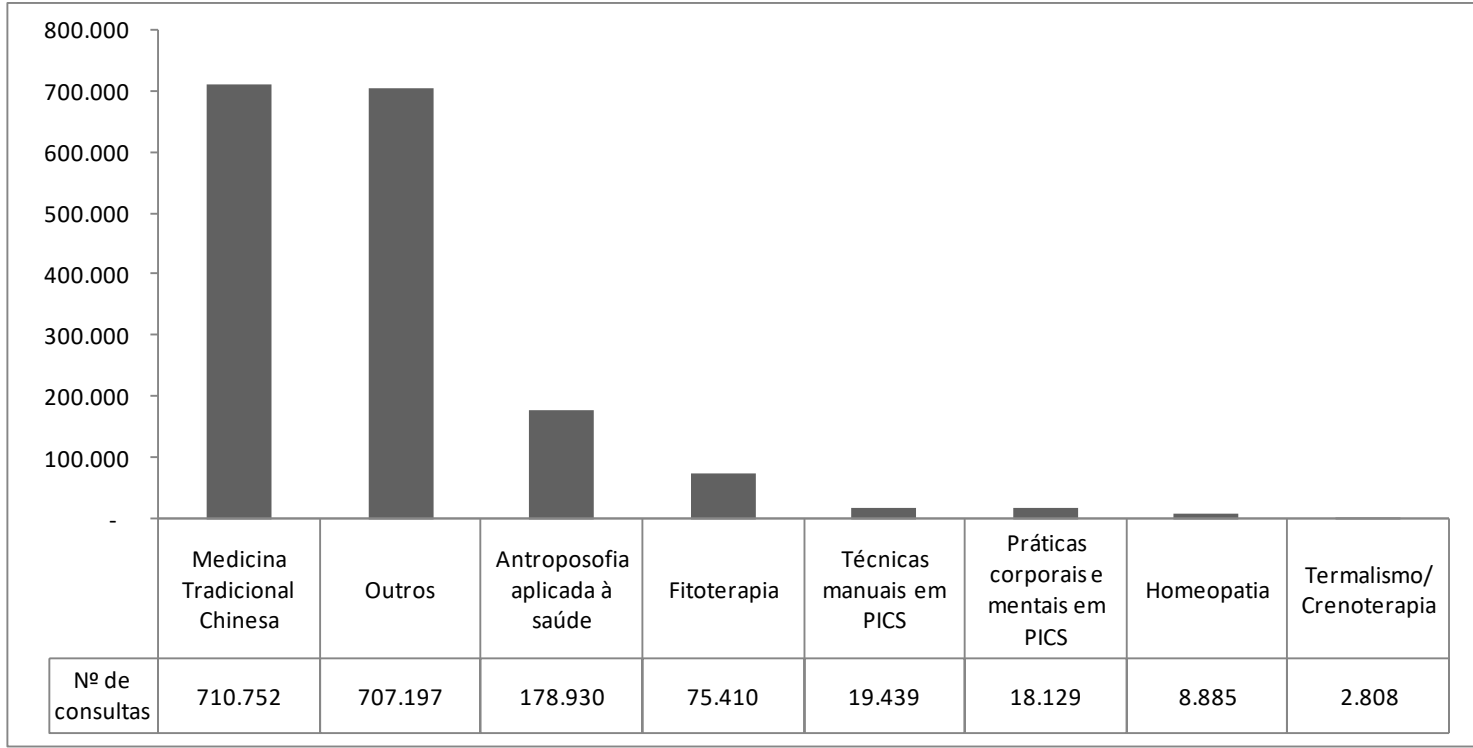

Fonte: e-SUS, DAB/SAS/MS (2016).

Figura 3. Número de participante por tipo de Curso EAD em PICS, Brasil, 2014-2016

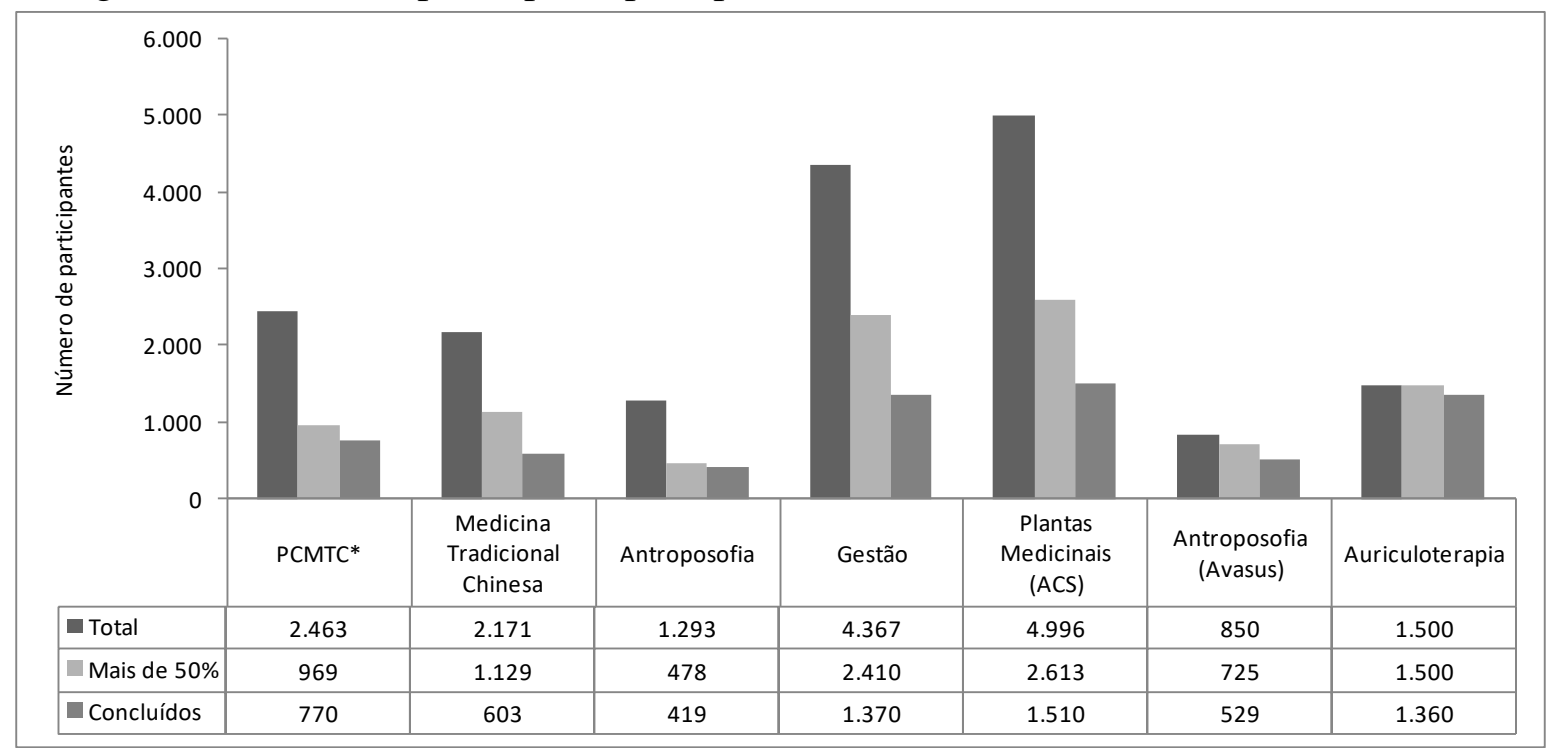

Fonte: DAB/MS (2016).

A pesquisa ainda não está bem estabelecida, e apenas uma parte dessas práticas e saberes acupuntura ${ }^{8}$, técnicas de meditação ${ }^{9,10}$, plantas medicinais e medicamentos dinamizados ${ }^{11,12},-$ tem sido estudados: sua eficácia e suas reações adversas. Ainda há muitas questões metodológicas e estruturais a ser desenvolvidas e que atendam as características dessas práticas para que as pesquisas sejam desenvolvidas em um padrão adequado, e na maioria dos trabalhos, as evidências ainda não são conclusivas. Isso certamente limita seu uso a doenças específicas, desconsiderando o potencial dessas práticas para promover a saúde em escalas populacionais ${ }^{7}$. 
ISSN 2179-6750

Apesar da polêmica e das dúvidas, é inegável e benéfica a aproximação dessas práticas, haja vista a sua natureza vitalista, que procura a cura e equilíbrio por meio de intervenções que reforçam ou induzem uma resposta natural do organismo ${ }^{13}$.

Com este enfoque no tripé - assistência, ensino (formação) e pesquisa -, que é a mesma da atuação universitária, a inserção das PICS no meio acadêmico poderá contribuir na intensificação destas práticas à política de promoção da saúde.

A Medicina Antroposófica (MA), abordagem integrativa destacada na presente experiência, foi introduzida no Brasil há mais de meio século mas ainda é pouco conhecida ${ }^{7}$. Apenas recentemente os sistemas nacionais de registro começaram a disponibilizar mecanismos para seu registro ${ }^{1,14}$

Atualmente, a MA é praticada em mais de 50 países, e é empregada no atendimento primário e secundário em mais de 20 países da União Europeia, aonde alcança um alto nível de satisfação entre os pacientes ${ }^{15}$. Em 2006, foi publicada a primeira revisão sistemática sobre a situação atual, eficácia, efetividade, segurança, utilização e custos da MA (Health Technology Assessment Report). A maioria dos trabalhos mostrou bons resultados, poucos efeitos colaterais, alta satisfação dos pacientes e boa relação custo/benefício, que foi atribuída à diminuição do número de internações hospitalares e do uso de medicamentos ${ }^{16}$. Kooreman e Baars ${ }^{17}$ mostraram que pacientes atendidos por médicos de família quer fossem homeopatas, acupunturistas ou antroposóficos sofreram menos internações, prescrição de drogas e os custos do cuidado foi menor.

A obstetrícia é outra área de intercessão de saberes no relato da presente experiência. Dentre as diferentes especialidades médicas, a saúde materno-infantil, particularmente, com relação à saúde obstétrica, vem sofrendo com a medicalização/intervenção cirúrgica excessiva. O enfoque mais fisiológico da gestação poderia ser resgatado com as racionalidades da medicina integrativa, proporcionando ensino, assistência e pesquisa, dentro de uma universidade, que é a detentora e divulgadora de novos conhecimentos, aprendizados e atuações.

A gravidez, sob o ponto de vista físico, mental e social é impactante, tanto para as mulheres quanto para seus familiares. Embora a gravidez na adolescência, socialmente problemática, tenha ocorrência frequente, a média de idade da primeira gestação vem crescendo progressivamente em nosso meio. Hoje, é comum, encontrar mulheres grávidas que já ultrapassaram a metade da terceira década de vida, considerada gestante idosa, nos livros clássicos de obstetrícia. Assim, essas grávidas começaram a apresentar mais frequentemente, doenças crônico-degenerativas como intercorrência durante esse período gestacional. Essa mudança atual no perfil das gestantes se defronta então com 
ISSN 2179-6750

uma prática clínica de maior complexidade, própria de uma doença crônica, incurável. Nesse tipo de pacientes, há um interesse cada vez maior de melhorar ao menos a sua qualidade de vida. Assim, o questionário genérico de qualidade de vida ${ }^{18}$ vem sendo empregado em inúmeros estudos e em diferentes populações. A definição de qualidade de vida, como bem coloca a Organização Mundial de Saúde, envolve diferentes domínios, sendo os principais, a saúde física e emocional, o funcionamento social e ambiental, a autonomia e os aspectos espirituais/religiosos ${ }^{19}$. Com esta visão mais abrangente do ser humano, contextualizado num ambiente socioeconomicocultural, fica mais fácil compreender que a necessidade da grávida ultrapassa os limites dos cuidados biológicos. A criança que está por vir, causará impacto na mulher enquanto gestante, em diversos domínios, desde recursos econômicos e sociais, como acesso aos serviços de saúde; impacto no desempenho das suas atribuições relacionadas ao trabalho tanto profissional quanto doméstico (limitação da capacidade funcional); assim como na saúde mental e sexual (mudança/acréscimo de papel na família).

Assim, de um lado, a crescente prevalência de doença crônico-degenerativa nas gestantes, e de outro lado, incentivo à qualidade de vida que proporcione menor complicação das doenças crônicas, pode-se, com a medicina integrativa, transdisciplinar, oferecer maiores oportunidades da grávida atingir o termo da gestação e levar para casa, um filho saudável. Deve ainda ser ressaltado que, durante a gestação, o potencial teratogênico de qualquer fármaco faz com que os profissionais optem muitas vezes por niilismo ou por métodos alternativos. A gravidez proporciona, mesmo em mulheres hígidas, inúmeros sintomas que podem interferir na sua qualidade de vida. Destes, ressalte-se a dor músculo-esquelética, que pode acometer em mais de $70 \%$ das gestantes ${ }^{8}$, assim como a fadiga, queixa muito comum no pré-natal, sendo a sua intensidade, maior no fim do dia, em relação ao período matutino, acometendo em até $60 \%$ das grávidas 20,21 . Dor, fadiga, stress, depressão e insônia, sintomas que impactam a qualidade de vida, podem trazer efeitos adversos sobre o porvir obstétrico e no concepto. A fadiga aumenta o risco de prematuridade $(\mathrm{OR}=3,45)^{22}$, assim como os sintomas depressivos $(R R=1,59){ }^{23}$. Dor musculoesquelética está associada com stress psicológico e estresse crônico psicofísico durante a gravidez ${ }^{24}$. Os estudos sobre crianças que foram expostas ao estresse crônico, principalmente durante o primeiro e segundo trimestres da gestação, mostram redução nas habilidades cognitivas, linguísticas e lúdicas com 2 e 5,5 anos de idade ${ }^{25-28}$. Quanto ao sono, Palagini et al. ${ }^{29}$, em sua revisão sistemática, encontraram associação entre perda crônica do sono com depressão pré-natal, diabetes gestacional, hipertensão arterial, préeclampsia/eclampsia, parto prematuro e inadequação do peso fetal. Com este panorama e ao mesmo tempo, desprovido de recurso farmacêutico seguro na gestação, vale pensar numa 
ISSN 2179-6750

perspectiva integrativa no cuidado da gestante. Além disso, os estudos da literatura apontam, tanto em doença crônica como numa gravidez de risco, a importância do apoio da família e amigos, para manter a continuidade biográfica, e portanto, oferecer uma abordagem sociopsicossomática da doença.

Desse modo, a assistência pré-natal conduzida com uma abordagem integrativa ampliada pode contribuir para o cuidado da mulher no período gestacional/puerperal e para um desfecho perinatal positivo.

\section{Métodos}

A Medicina Antroposófica, (etmologicamente vem do grego anthropos: homem e sophia: sabedoria) idealizada por Rudolf Steiner, cuida do paciente integralmente, no seu corpo, alma e espírito, atuando através de tratamento medicamentoso (mineral, vegetal e animal), introduzindo formas de modificação de hábitos evidenciados pela anamnese complementada pela biografia (tomar consciência dos comportamentos prejudiciais e mudá-los, como uma das ferramentas utilizadas pela psicologia) e outras condutas ativas como orientação nutricional, terapias externas corporais como hidroterapia/escalda-pé/ pernas e massagem rítmica com técnica de deslizamento ${ }^{30}$. Com este modelo de racionalidade médica, foi proposto o acompanhamento pré-natal antroposófico, isto é, uma abordagem terapêutica multimodal e integral, na Universidade Federal de São Paulo, em 2007. Nasce o Projeto NUMA: Núcleo de Medicina Antroposófica (Quadro 1). 


\section{Quadro 1. Atendimento NUMA}

O Núcleo de Medicina Antroposófica (NUMA) do Departamento de Obstetrícia da Universidade Federal de São Paulo é um projeto que engloba a pesquisa, o ensino e a assistência.

O ambulatório do NUMA faz o acompanhamento de mulheres no período pré-natal e no puerpério. Este serviço, cuja atividade ocorre uma vez por semana, é destinado para grávidas de risco habitual; no entanto, por pertencer a um hospital universitário (terciário), é frequente ter encaminhamentos de pacientes portadoras de doenças crônicas (como esclerose múltipla, epilepsia, drogadicção, vaginismo etc.), que estavam sendo acompanhadas nos ambulatórios de especialidades. A equipe multidisciplinar compreende profissionais médicos (obstetras, clínico, dermatologista, homeopata, acupunturista), fisioterapeutas, enfermeiras, nutricionista e psicólogos treinados no sistema de tratamento antroposófico. Esta equipe atende segundo a rotina de atendimento do NUMA, harmoniosamente pré-estabelecida pelos coordenadores de cada entidade profissional. No atendimento inicial, é realizada consulta por médico obstetra, dermatologista e avaliação psicológica, preenchendo a Ficha Clínica Antroposófica.

$\mathrm{Na}$ segunda consulta, são realizadas avaliações adicionais por fisioterapeuta, nutricionista e por enfermeira Caso haja alguma intercorrência que demande outros profissionais, estes são acionados.

A partir da segunda consulta, a paciente participa de um programa educacional, e também os acompanhamentos específicos/intervenção terapêutica (questionário de qualidade de vida-SF36, questionário de Beck de depressão e de ansiedade, biografia, contos de fadas, desenho, orientação alimentar, terapias externas como escalda-pés, massagens, e cinesioterapia). que foram indicados após a avaliação inicial.

Os retornos de consulta médica obedecem a uma rotina mensal até 32 semanas, quinzenal até 35 semanas e semanal até o parto; com possibilidade de antecipação da agenda caso clinicamente necessário. Com o início do retorno semanal, no terceiro trimestre, inicia-se o preparo para o parto: biológico (conhecer sintomas e autocuidado), psicológico (empoderamento e senso de coerência) e fisioterapêutico (do assoalho pélvico). O parto e os cuidados do pós-parto imediato não são realizados por esta equipe, e sim pelos profissionais de plantão hospitalar. Após o nascimento e alta hospitalar, a puérpera tem orientação quanto ao aleitamento pelas enfermeiras, de readequação da rotina diária/papel de mãe-esposa-profissional pelos psicólogos, de vida sexual/ anticoncepção pelos obstetras no retorno ambulatorial. 


\section{Resultados}

A aprovação da PNPIC impulsionou a criação do Núcleo de Medicina Antroposófica da Escola Paulista de Medicina/Universidade Federal de São Paulo (UNIFESP), no contexto do Observatório da $\mathrm{MA}^{31}$, contando com incentivo de organizações do terceiro setor para o início de suas atividades. Em 2011, foi criado o NUMEPI, Núcleo de Medicina e Práticas e Integrativas que agrega várias racionalidades médicas e cuidados integrativos, vinculado à Pró-Reitoria de Extensão. Pelo fato da UNIFESP ser um centro de pesquisa, ensino e assistência, seu primeiro impulso foi focado na pesquisa com as gestantes, uma vez que, até o início de 2007, a literatura científica (Pubmed) havia publicado apenas 47 artigos, com a palavra-chave "antroposophic medicine", mas nenhum estudo com as gestantes. A Medicina Antroposófica era pouco conhecida no meio acadêmico; assim, não despertou interesse nos alunos do curso de pós-graduação senso estrito. Constatado esse fato, a partir de 2009, a coordenação se empenhou em organização de cursos (ensino), de onde se originaram alunos interessados em pesquisa na área. Assim, o tripé universitário ensino-pesquisa-assistência tem garantido a sustentabilidade do projeto. Vale ressaltar que a assistência (pré-natal) tem sido fundamental, tanto para o ensino como para a pesquisa. Assim, o atendimento foi sendo estruturado ao longo desses dez anos com equipe multiprofissional, com linguagem antroposófica transdisciplinar, de forma a uniformizar e somar os efeitos positivos das diferentes abordagens terapêuticas, no formato de discussão de casos clínicos onde todos os profissionais participam (Quadro 1). Esta linguagem foi padronizada pelo NUMA com anamnese antroposófica, incorporando os conceitos de bi, tri, tetra, heptamembração, doze sentidos e biografia 32. Além disso, as gestantes são convidadas a participar do programa educativo semanal com foco salutogenético (Quadro 2).

Quadro 2. Palestras sobre orientações e cuidados salutogenéticos

\section{Cronograma: 4 ciclos anuais com 8 temas (semanais):}

1. Assistência pré-natal

2. Assistência ao parto

3. Assistência ao puerpério e aleitamento

4. Aspectos nutricionais

5. Sexualidade no ciclo gravídicopuerperal

6. Aspectos emocionais e maternagem

7. Cuidados estéticos/dermatológico

8. Atividade física / fisioterapia 
Com esta rotina mais organizada nos últimos sete anos, a média anual de atendimento tem sido no total, em torno de 1.450, de consultas médicas, psicológicas, fisioterapêuticas, nutricionais e de enfermagem.

No que diz respeito ao ensino, foram realizados nove cursos de extensão: antroposofia na saúde (3), semiologia antroposófica (4), doze sentidos (1) e a cura do ferimento (1) frequentados por mais de 400 graduandos/profissionais. Quanto à especialização, em 2017, o projeto NUMA está na vigência do terceiro curso com atividade prática no ambulatório; ou seja, até 2016 foram formados mais de 50 especialistas em antroposofia na saúde. O conteúdo programático está resumido no Quadro 3.

Desde 2012, o ambulatório do NUMA é estágio oficial obrigatório de Residência Médica (em Medicina de Família e Acupuntura), da Residência Multiprofissional (Fisioterapia/Psicologia/Enfermagem em Saúde da Mulher) e da Graduação em Medicina (sexto ano). Além dos cursos, a divulgação da medicina antroposófica na UNIFESP também ocorreu através de congresso (2014), simpósio internacional em parceria com a Associação Brasileira de Medicina Antroposófica (ABMA) (2016) e jornadas (2014 e 2016), a mais de 300 pessoas. Está em curso também um intercâmbio internacional com a Filderklinik, um hospital antroposófico de Stuttgart, e já tivemos duas médicas residentes em Ginecologia e Obstetrícia (2011: uma enviada e outra recebida).

Quanto à pesquisa, os medicamentos antroposóficos (Viscum album e Bryophyllum) e a atitude profissional da equipe médica renderam duas teses de doutorado e três de mestrado, que por sua vez, foram a base dos dois artigos originais publicados em revistas científicas de impacto ${ }^{11,33}$. Somados a essa produção científica, alguns relatos de casos foram apresentados em congressos médicos nacionais/internacionais, e trabalhos de conclusão do curso dos alunos de especialização publicados em revista de menor impacto. 
Quadro 3. Cronograma do curso de especialização "Antroposofia na Saúde"

Carga horária: 372 horas/aula (50\% teórica e $26 \%$ prática e $24 \%$ atividades práticas sensório-expressivas)

\section{Aulas teóricas:}

1. Conceito de medicina integrativa

2. Doente ou doença?

3. Salutogênese e Medicina Antroposófica

4. O ser terapeuta segundo Filon de Alexandria

5. Fundamentos filosóficos da Medicina Antroposófica

6. Fenomenologia de Goethe: observação da natureza e do ser humano

7. Empatia clínica

8. Gravidez, parto, puerpério e aleitamento do ponto de vista antroposófico

9. Metodologia científica, didática e projeto de Trabalho de Conclusão de Curso

10. Constituição polar: inflamação e esclerose; histeria e neurastenia

11. Quadrimembração e os temperamentos

12. Trimembração e senso de coerência

13. Biografia

14. Heptamembração

15. Doze sentidos

16. Farmácia antroposófica

17. Nutrologia

18. Experiencias de profissionais/instituições antroposóficas

\section{Prática ambulatorial}

1.1.Acompanhamento da assistência

1.2.Discussões de casos clínicos

\section{Atividades sensório-expressivas}

1. Contos

1.1. Contos tradicionais: A longa viagem

1.2.Narrativas bíblicas: Tobias e o Arcanjo Rafael

1.3. Contos de fadas: A chave de ouro, Os músicos de Bremen, Os seis cisnes, João o Fiel, A moça sem mãos, e outros.

2. Terapia artística

2.1.Desenho de forma

2.2.Pintura: aquarela, pastel seco, giz de cera

2.3.Argila

3. Contemplações de obras de arte

3.1.Medida áurea

3.2. Arte como reflexo da evolução da consciência

4. Euritmia

5. Cantoterapia

6. Massagem ritmica

7. Terapias externas antroposóficas

8. Estudo biográfico de personalidades

9. Seis exercícios básicos de Rudolf Steiner

10. Visita ao laboratório farmacêutico antroposófico

11. Reorganizção Neuro-funcional: método Padovan

12. Sentidos humanos 


\section{Discussão}

A escola de medicina com racionalidade médica convencional, biomédica, acaba sendo desafiada quando se propõe a Medicina Integrativa. A instituição organizada segundo critérios de hierarquia (verticalização do poder) está sendo confrontada por parceria (horizontalização e troca), tanto na relação médico-paciente como entre as diversas categorias de profissão. Além disso, o receio do erro médico leva quase sempre, à busca de evidências científicas, que reforça cada vez mais ao conceito de doença e menos à relação com o doente. Poder-se-ia dizer, então, que a implantação de MTC numa universidade não é elementar. Por outro lado, o tripé assistência-ensinopesquisa das universidades pode contribuir sobremaneira na sustentabilidade da MTC. Ao ensinar os profissionais, estes darão assistência à população; e se nessa atuação perceber algo que possa ser melhorado, pesquisas poderão ser realizadas para seu aprimoramento. O modelo clássico de assistência à saúde focado somente no papel do médico vem sendo questionado há algum tempo. Já em 1998, o pensamento transdisciplinar já era tema na ciência social de saúde ${ }^{34}$. Este pensamento está ligado à teoria da complexidade: relação não linear, causalidade interativa e propriedades emergentes de sistemas. Mesmo assim, este modelo ainda é pouco adotado nas unidades de serviços de saúde. Na saúde materno-infantil, onde a criança necessita de higiene social, mental e biológico para o seu crescimento e desenvolvimento, a transdisciplinaridade é mais enfatizada ${ }^{35}$. A atuação multiprofissional já no período perinatal possibilita promoção de saúde infantil, evitando que esta criança seja vítima de abuso ou negligência; nesse caso, sua recuperação pode necessitar também cuidado transdisciplinar ${ }^{36}$.

A abordagem multimodal antroposófica permite que a gestante confidencie não apenas os sintomas físicos, mas também os psíquicos; a importância de compartilhar fatos biográficos tem sido fundamental na promoção da relação profissionais da saúde-paciente. Aumenta a confiança da paciente em relação ao serviço e maior aderência ao tratamento preconizado. No estudo de Carvalho ${ }^{37}$ a medida do calor humano dos médicos do ambulatório $\left(25,5 \pm 6{ }^{\circ} \mathrm{C}\right)$ foi muito próxima da do médico ideal $\left(27,3 \pm 4{ }^{\circ} \mathrm{C}\right)$, e de pessoas carinhosas $\left(28,2 \pm 3{ }^{\circ} \mathrm{C}\right)$, e mais altas do que pessoas medianamente ternas $\left(18,8 \pm 7^{\circ} \mathrm{C}\right)$. Além disso, a aplicação de questionário de senso de coerência de Antonovsky ${ }^{38}$ e o detalhamento biográfico dos fatos da vida pela equipe de psicologia faz com que as gestantes se apoderem da sua capacidade de resiliência. A resiliência é fundamental para o enfrentamento do parto, esse importante fator de aflição para a maioria das gestantes; assim como do puerpério, na perseverança de lidar diariamente com recém-nascido, esse desconhecido que chora de fome e sede, chora com o desconforto das vestes molhadas de urina, sujas de fezes ou ainda, que se mostra insatisfeito com qualquer coisa que incomode o seu bem estar. 
ISSN 2179-6750

A não atuação da equipe durante a assistência ao parto é ainda um ponto fraco da nossa atividade. Considerando que o parto é um evento fisiológico e passível de ocorrer a qualquer momento, deveria disponibilizar profissionais 24 horas por dia e sete dias da semana. Esse serviço não é oferecido por impossibilidade conjuntural, mas no nosso programa cumprimos a finalidade pré-natal de orientação e estímulo do vínculo com o local de parto. As gestantes são orientadas a procurar as maternidades do SUS. É objetivo do NUMA que as parturientes se dirigirem aos hospitais, mais confiantes em si mesmas. Todo o processo da parturição e os métodos não farmacológicos de dor são ensinados durante o pré-natal às gestantes e seus acompanhantes, quando presentes. Assim, estando o estresse sob controle, a ocitocina endógena ${ }^{39}$ desempenhará melhor seu papel, tanto na parturição, como no aleitamento e vínculo materno-infantil.

Durante o pré-natal também é abordada a questão da episiotomia, que é uma intervenção temida pelas gestantes. Quando sistemática, é até mesmo considerada por alguns, como violência obstétrica. O estudo do assoalho pélvico possibilitou não só dimensionar a sua extensibilidade (com epi-no®) ${ }^{40}$, como também, juntamente com a massagem, aumentá-la a tal ponto de contraindicar a episiotomia e/ou reduzir o risco de laceração perineal na passagem do feto.

No que diz respeito ao ensino, onde mais de $40 \%$ das atividades se concentram nos finais de semana; portanto, com dez horas corridas por dois dias consecutivos, é difícil imaginar rendimento na aprendizagem somente com aulas expositivas e teóricas. Segundo Ross e Lypson ${ }^{41}$, o trabalho com estudantes de medicina usando apresentação narrativo-artística das pacientes, mostrou que esta forma de abordagem levou os estudantes à reflexão, ajudando-os à maior compreensão dos viezes da conduta médica no tratamento da dor. Eles também tiveram uma visão mais empática das pacientes como indivíduos, e reconheceram como as crenças dos médicos podem levar a disparidades no cuidado à saúde. Os autores concluem que a combinação de métodos - arte, narrativa e reflexão escrita ajudaram os estudantes a compreender os seus próprios viezes que poderiam influenciar no cuidado ao paciente. Entendemos que as diversas formas de arte são muito ricas na expressão da individualidade; isto permite que se faça uma reflexão e autoanálise/autoconhecimento. Assim, a arte pode ser o instrumento com poder transformador da individualidade humana. Este conceito fez com que a metade da atividade prática do curso fosse artística.

Com relação à pesquisa em laboratório, a segurança do uso medicamentoso de Bryophyllum durante a gestação foi confirmada em estudos experimentais ${ }^{11}$. Também foi demonstrado que suas folhas utilizadas para suco ou chá não continham substâncias cardiotóxicas como o bufadienolídeo, segundo a tese de Pereira ${ }^{42}$. Como mostra o trabalho de Furer et al. ${ }^{43}$, este medicamento é prescrito 
ISSN 2179-6750

mais para gestantes do que para pacientes ginecológicas, sendo avaliado muito positivamente como uterolítico: a prematuridade é hoje, um dos grandes problemas em obstetrícia.

Como palavras finais, este modelo de trabalho abre a possibilidade de reduzir o risco de burnout, estresse profissional do médico obstetra. Dividir responsabilidade com outros profissionais, receber maior confiança das pacientes com melhor relação médico-paciente e menor complicação pela maior adesão às recomendações médicas/melhor qualidade de vida podem ser os pilares do desfecho obstétrico feliz e maior satisfação das usuárias.

\section{Conclusão}

Embora a PNPIC não tenha ainda representado uma fonte de incentivo financeiro capaz de contribuir para a sustentabilidade de experiências exitosas no SUS, ela representa um importante marco político normativo para a abertura de espaços estratégicos em serviços e Instituições de Ensino e Pesquisa, além de despertar o interesse e legitimar a busca pela mudança do modelo de cuidado, tanto do usuário quanto dos profissionais de saúde.

O crescente descontentamento no sistema de saúde atual nos instiga à mudança de paradigma; isso também ocorre na área de obstetrícia. A abordagem da Antroposofia na área da saúde compreende um modelo integrativo baseado no cuidado multiprofissional com valorização da pessoa como protagonista do seu processo e ponto de convergência das intervenções. O modelo de pré-natal multimodal orientado pela Antroposofia, praticado no NUMA tem atraído muitos profissionais e usuários que buscam a ampliação de seus saberes e a aquisição de novos instrumentos para o aprimoramento de sua prática profissional, humanização e satisfação com os resultados alcançados. Além disso, tem representado importante ponto de formação e produção do conhecimento técnico científico. Este modelo de trabalho apresentado pelo NUMA é uma proposta que pode responder a alguns dos dilemas dessa especialidade.

\section{Agradecimentos}

O NUMA-UNIFESP agradece o apoio recebido dos Profissionais de Saúde que atuam no serviço, ao Instituto Mahle e à Associação Brasileira de Medicina Antroposófica - ABMA.

Conflito de interesse: os autores declaram não ter conflito de interesse. 


\section{Referências}

1. Sousa IMC, Tesser CD. Medicina Tradicional e Complementar no Brasil: inserção no Sistema Único de Saúde e integração com a atenção primária. Cad Saude Publica. 2017;33(1):e00150215. https://doi.org/10.1590/0102-311X00150215.

2. World Health Organization. WHO global strategy on traditional and alternative medicine. Public Health Rep. 2002;117(3):300-1.

3. World Health Organization. WHO traditional medicine strategy: 2014-2023. [Hong Kong]: WHO; 2013. $76 \mathrm{p}$.

4. Tesser CD, Barros NF. Medicalização social e medicina alternativa e complementar: pluralização terapêutica do Sistema Único de Saúde. Rev Saude Publica. 2008;42(5):914-20. http://dx.doi.org/10.1590/S0034-89102008000500018.

5. Ministério da Saúde, Secretaria de Atenção à Saúde, Departamento de Atenção Básica. Política Nacional de Práticas Integrativas e Complementares no SUS. Brasília, DF: Ministério da Saúde; 2006 [acesso 24 jun 2017]. Disponível em: http://dab.saude.gov.br/portaldab/pnpic.php

6. Brasil, Ministério da Saúde. Portaria No 849, de 27 de março de 2017. Inclui a Arteterapia, Ayurveda, Biodança, Dança Circular, Meditação, Musicoterapia, Naturopatia, Osteopatia, Quiropraxia, Reflexoterapia, Reiki, Shantala, Terapia Comunitária Integrativa e Yoga à Política Nacional de Práticas Integrativas e Complementares. Brasília, DF: Diário Oficial da União; 28 mar. 2017.

7. Sousa IMC, Bodstein RCA, Tesser CD, Santos FAS, Hortale VA. Práticas integrativas e complementares: oferta e produção de atendimentos no SUS e em municípios selecionados. Cad Saude Publica. 2012;28(11):2143-54. http://dx.doi.org/10.1590/S0102$311 X 2012001100014$.

8. Silva JBG, Nakamura MU, Cordeiro JA, Kulay Jr. L. Acupuncture for low back pain in pregnancy--a prospective, quasi-randomised, controlled study. Acupunct Med 2004;22(2):607. http://dx.doi.org/10.1136/aim.22.2.60.

9. Cardoso R, Souza E, Camano L, Leite JR. Meditation in health: an operational definition. Brain Res Brain Res Protoc. 2004 Nov;14(1):58-60. https://doi.org/10.1016/j.brainresprot.2004.09.002. 10. Byrne J, Hauck Y, Fisher C, Bayes S, Schutze R. Effectiveness of a Mindfulness-Based Childbirth Education pilot study on maternal self-efficacy and fear of childbirth. J Midwifery Womens Health. 2014 Mar-Apr;59(2):192-7. https://doi.org/10.1111/jmwh.12075. Epub 2013 Dec 10. 
ISSN 2179-6750

11. Hosomi JK, Ghelman R, Quintino MP, Souza E, Nakamura MU, Moron AF. Effects of chronic Bryophyllum pinnatum administration on wistar rat pregnancy. Forsch Komplementmed 2014;21(3):184-9. https://doi.org/10.1159/000363709. Epub 2014 Jun 16.

12. Boltman-Binkowski H. A systematic review: Are herbal and homeopathic remedies used during pregnancy safe? Curationis 2016 Apr 13;39(1):1514. https://doi.org/10.4102/curationis.v39i1.1514.

13. Queiroz MS. O itinerário rumo às medicinas alternativas: uma análise em representações sociais de profissionais da saúde. Cad Saude Publica. 2000 abr-jun;16(2):363-75.

14. Simoni C, Antonini CD, Benevides I, Brina NT. Observatório dos serviços de medicina antroposófica no Sistema Único de Saúde: resultados parciais do monitoramento no período 2009-2011. Arte Med Ampl. 2012;32(1):26-34.

15. Esch BM, Marian F, Busato A, Heusser P. Patient satisfaction with primary care: an observational study comparing anthroposophic and conventional care. Health Qual Life Outcomes. 2008 Sep 30;6:74-89. https://doi.org/10.1186/1477-7525-6-74.

16. Follador ECR. Medicina Antroposófica: um novo paradigma para as questões da medicina moderna. Rev Med (Sao Paulo). 2013;92(3):166-72. http://dx.doi.org/10.11606/issn.16799836.v92i3p166-172.

17. Kooreman P, Baars EW. Patients whose GP knows complementary medicine tend to have lower costs and live longer. Eur J Health Econ. 2012 Dec;13(6):769-76. http://dx.doi.org/10.1007/s10198-011-0330-2. Epub 2011 Jun 22.

18. Ciconelli RM, Ferraz MB, Santos W, Meinão I, Quaresma MR. Tradução para a língua portuguesa e validação do questionário genérico de qualidade de vida SF-36 (Brasil SF-36). Rev Bras Reumatol. 1999;39(3):143-50.

19. The WHOQOL Group. The World Health Organization Quality of Life assessment (WHOQOL): position paper from the World Health Organization. [s.1.]: WHOQOL; 1995.

20. Tsai SY, Lin JW, Kuo LT, Thomas KA. Daily sleep and fatigue characteristics in nulliparous women during the third trimester of pregnancy. Sleep. 2012 Feb 1;35(2):257-62. http://dx.doi.org/ http://dx.doi.org/10.5665/sleep.1634.

21. Gay CL, Lee KA, Lee SY. Sleep patterns and fatigue in new mothers and fathers. Biol Res Nurs. 2004 Apr;5(4):311-8. https://doi.org/10.1177/1099800403262142.

22. Ko YL, Wu YC, Chang PC. Physical and social predictors for pre-term births and low birth weight infants in Taiwan. J Nurs Res. 2002 Jun;10(2):83-9. http://dx.doi.org/10.1097/01.JNR.0000347586.08328.d6. 
ISSN 2179-6750

23. Orr ST, James SA, Blackmore Prince C. Maternal prenatal depressive symptoms and spontaneous preterm births among African-American women in Baltimore, Maryland. Am J Epidemiol. 2002 Nov 1;156(9):797-802.

24. Bakker EC, van Nimwegen-Matzinger CW, Ekkel-van der Voorden W, Nijkamp MD, Völlink T. Psychological determinants of pregnancy-related lumbopelvic pain: a prospective cohort study. Acta Obstet Gynecol Scand. 2013 Jul;92(7):797-803. http://dx.doi.org/10.1111/aogs.12131. Epub 2013 Mar 29.

25. Charil A, Laplante DP, Vaillancourt C, King S. Prenatal stress and brain development. Brain Res Ver. 2010 Oct 5;65(1):56-79.

26. King S, Laplante DP. The effects of prenatal maternal stress on children's cognitive development: Project Ice Storm. Stress. 2005 Mar;8(1):35-45.

27. Laplante DP, Barr RG, Brunet A, Galbaud du Fort G, Meaney ML, Saucier JF, et al. Stress during pregnancy affects general intellectual and language functioning in human toddlers. Pediatr Res. 2004 Sep;56(3):400-10. http://dx.doi.org/10.1203/01.PDR.0000136281.34035.44.

28. Laplante DP, Brunet A, Schmitz N, Ciampi A, King S. Project Ice Storm: prenatal maternal stress affects cognitive and linguistic functioning in 5 1/2-year-old children. J Am Acad Child Adolesc Psychiatry. 2008 Sep;47(9):1063-72. http://dx.doi.org/10.1097/CHI.0b013e31817eec80.

29. Palagini L, Gemignani A, Banti S, Manconi M, Mauri M, Riemann D. Chronic sleep loss during pregnancy as a determinant of stress: impact on pregnancy outcome. Sleep Med. 2014 Aug;15(8):853-59. http://dx.doi.org/10.1016/j.sleep.2014.02.013. Epub 2014 May 27.

30. Nakamura MU, Ghelman R. Medicina antroposófica e obstetrícia. In: Moron AF, Camano L, Kulay Jr. L, editors. Obstetrícia. 1a ed. Barueri: Editora Manole; 2011. p. 271-87.

31. Benevides I. Inserção da medicina antroposófica no Sistema Único de Saúde: aspectos históricos, marcos normativos e desafios para sua implementação. Arte Med Ampl. 2012 jan$\operatorname{mar} ; 32(1): 4-11$.

32. Ghelman R, Hosomi JK, Yaari M, Castro AV, Pravatto Jr M, Costa LAN, et al. Ficha clínica antroposófica do Núcleo de Medicina Antroposófica da Universidade Federal de São Paulo Escola Paulista de Medicina. Arte Méd Ampl. 2012 jan-mar;32(1):12-21.

33. Facina AS, Facina G, Silva IDCG, Gonçalves GA, Almeida FA, Noronha SAAC, et al. Viscum Album Modulates Apoptotic Related Genes in Melanoma Tumor of Mice. Am J Mol Biol. 2014;4(2):49-58. http://dx.doi.org/10.4236/ajmb.2014.42007. 
ISSN 2179-6750

34. Albrecht G, Freeman S, Higginbotham N. Complexity and human health: the case for a transdisciplinary paradigm. Cult Med Psychiatry. 1998 Mar;22(1):55-92.

35. Terhaar M, O'Keefe S. A new advanced practice role focused on outcomes management in women's and children's health. J Perinat Neonatal Nurs. 1995 Dec;9(3):10-21.

36. Hyter YD, Atchison B, Henry J, Sloane M, Black-Pond C. A response to traumatized children: developing a best practices model. Occup Ther Health Care. 2002;15(3-4):113-40. https://doi.org/10.1080/J003v15n03_07.

37. Carvalho MSM. Tradução e validação do questionário de empatia "warmometer" para o português brasileiro [Tese]. [São Paulo]: Universidade Federal de São Paulo; 2016.

38. Antonovsky A. The structure and properties of the sense of coherence scale. Soc Sci Med. 1993 Mar;36(6):725-33.

39. Unternaehrer E, Bolten M, Nast I, Staehli S, Meyer A H, Dempster E, et al. Maternal adversities during pregnancy and cord blood oxytocin receptor (OXTR) DNA methylation. Soc Cogn Affect Neurosci. 2016 Sep;11(9):1460-70. https://doi.org/10.1093/scan/nsw051. Epub 2016 Apr 22.

40. Zanetti MR, Petricelli CD, Alexandre SM, Paschoal A, Araujo Júnior E, Nakamura MU. Determination of a cutoff value for pelvic floor distensibility using the Epi-no balloon to predict perineal integrity in vaginal delivery: ROC curve analysis. Prospective observational single cohort study. Sao Paulo Med J. 2016;134(2):97-102. https://doi.org/10.1590/15163180.2014.8581009. Epub 2015 Mar 17.

41. Ross PT, Lypson ML. Using artistic-narrative to stimulate reflection on physician bias. Teach Learn Med. 2014;26(4):344-9. https://doi.org/10.1080/10401334.2014.945032. 42. Pereira KMF. Identificação e sazonalidade dos constituintes de Kalanchoe pinnata (Lans) Pers. - planta com ação uterolítica e ansiolítica [Tese]. [São Paulo]: Universidade Federal de São Paulo; 2016.

43. Fürer K, Simões-Wüst AP, Winkler A, Amsler N, Schnelle M, von Mandach U. The Application of Bryophyllum pinnatum Preparations in Obstetrics and Gynaecology - a Multicenter, Prospective Observational Study. Forsch Komplementmed 2015;22(4):231-6. https://doi.org/10.1159/000437154. Epub 2015 Aug 3. [German]. 